\title{
An experimentally validated parametrically excited vibration energy harvester with time-varying stiffness
}

\author{
Bahareh Zaghari $^{a}$, Emiliano Rustighi ${ }^{a}$ and Maryam Ghandchi Tehrani ${ }^{a}$ \\ ${ }^{a}$ University of Southampton, Highfield Campus, Southampton, UK
}

\begin{abstract}
Vibration energy harvesting is the transformation of vibration energy to electrical energy. The motivation of this work is to use vibration energy harvesting to power wireless sensors that could be used in inaccessible or hostile environments to transmit information for condition health monitoring. Although considerable work has been done in the area of energy harvesting, there is still a demand for making a robust and small vibration energy harvesters from random excitations in a real environment that can produce a reliable amount of energy. Parametrically excited harvesters can have time-varying stiffness. Parametric amplification is used to tune vibration energy harvesters to maximize energy gains at system superharmonics, often at twice the first natural frequency. In this paper the parametrically excited harvester with cubic and cubic parametric nonlinearity is introduced as a novel work. The advantages of having cubic and cubic nonlinearity are explained theoretically and experimentally.
\end{abstract}

Keywords: Parametrically excited harvester, condition health monitoring, cubic and cubic parametric nonlinearity, stability

\section{INTRODUCTION}

In recent years, different vibration-based energy harvesting methods have been introduced. ${ }^{1,2}$ The motivation of this work is to use vibration energy harvesting to power wireless sensors that could be used in inaccessible or hostile environments to transmit information for condition health monitoring. Linear vibration energy harvesters are based on a vibrating mechanical structure, usually with additional seismic mass, where the driving force is applied parallel to the direction of the oscillatory displacement. In linear single-degree-of-freedom (SDOF) systems, the maximum displacement occurs at the resonance frequency, hence linear harvesters generate the most energy when the natural frequency of the device is equal to the excitation frequency. Consequently, a small change in the excitation frequency can reduce the amount of harvested power. This restricts the development and performance of linear harvesters. In order to improve the performance of existing harvesters, different methods for tuning and broadening the bandwidth of the harvester have been proposed. ${ }^{3-6}$

A physical system subject to an external load which appears in the equation of motion in the form of periodic time-variant parameter can be considered as a parametrically excited system. ${ }^{7}$ Parametric excitation has been observed in cable-stayed bridges due to support, deck or tower motions. ${ }^{8}$ Energy harvesting from directly-excited non-parametric systems has been studied extensively, but not much work has been done in exploiting parametric excitation in the context of vibration energy harvesting. ${ }^{9-12}$ Jia et al. ${ }^{13}$ investigated the use of parametric excitation in MEMS vibration energy harvesters in order to increase the power density which is limited by damping. An increase in the power density and frequency bandwidth for the parametrically excited MEMS vibration energy harvester was achieved. However, despite a careful design, a damping-dependent initiation threshold was still a limiting factor.

In this paper, the dynamic behavior of a parametrically excited beam is considered and its potential exploitation for the problem of energy harvesting is investigated. This system can be a representative for real structures under parametric resonance, such as cable-stayed bridges or marine flexible risers. The cubic and cubic parametric nonlinearity effect in parametrically excited system is explained analytically and experimentally. The advantage of having nonlinearity in order to reduce the unbounded amplitude of the linear Mathieu equation

Further author information: (Send correspondence to Bahareh Zaghari)

Bahareh Zaghari: E-mail: b.zaghari@soton.ac.uk, Telephone: +44(0)2380592294

Smart Materials and Nondestructive Evaluation for Energy Systems 2015, edited by Norbert G. Meyendorf, Proc. of SPIE Vol. 9439, 94390S · @ 2015 SPIE · CCC code: 0277-786X/15/\$18 · doi: 10.1117/12.2084219 
is shown in the experimental results. The system only has decaying and steady-state responses when cubic and cubic nonlinearity exists. The free response study of the nonlinear parametrically excited system has been mentioned in few work, although the forced responses were explained in several publications. ${ }^{14-16}$ The voltage across the optimum resistive load parallel with the piezoelectric sensor is measured to find the average dissipated power. The Root Mean Square power (RMS power) for a parametrically excited harvester at different frequency is compared.

\section{METHODOLOGY}

\subsection{The method of averaging for solving a nonlinear system with a time-varying coefficient}

The governing nonlinear parametrically exited differential equation is

$$
m_{\mathrm{t}} \ddot{z}+\varepsilon c_{\mathrm{t}} \dot{z}+\left(k_{1}+\varepsilon k_{\mathrm{p} 1} \cos (\Omega t)\right) z+\varepsilon k_{3} z^{3}+\varepsilon k_{\mathrm{p} 3} \cos (\Omega t) z^{3}=0,
$$

where $z(t)$ is the mass displacement, $m_{\mathrm{t}}$ is the total mass, $c_{\mathrm{t}}$ is the total viscous damping coefficient, $\varepsilon$ is the bookmark term, $k_{1}$ is the constant stiffness, $k_{\mathrm{p} 1}$ is the parametric stiffness with the parametric frequency $\Omega, k_{3}$ is the cubic stiffness, and $k_{\mathrm{p} 3}$ is the cubic parametric stiffness. This equation represent a parametrically exited system with nonlinear terms. Eq. (1) is known as nonlinear Mathieu equation, and has been solved analytically with different methods such as multiple scales and the method of averaging. ${ }^{7}$ Here the method of averaging is implemented based on previous work. ${ }^{7,17,18}$ The method of averaging is very sensitive to the $\varepsilon$ value; for small values of $\varepsilon$, which means small value of parametric and nonlinear terms, this method solves the nonlinear Mathieu equation accurately. In this paper, this method is used for a large $\varepsilon$, and the result is verified with a numerical method. Normalizing Eq. (1) with the total mass $m_{t}$ yields

$$
\ddot{z}+2 \varepsilon \zeta \omega_{n} \dot{z}+\omega_{n}^{2}(1+\varepsilon \delta \cos (\Omega t)) z+\omega_{n}^{2}(\varepsilon \alpha+\varepsilon \gamma \cos (\Omega t)) z^{3}=0,
$$

where $\delta=\frac{k_{\mathrm{p} 1}}{k_{1}}, \alpha=\frac{k_{3}}{k_{1}}$, and $\gamma=\frac{k_{\mathrm{p} 3}}{k_{1}}$. Eq. (2) is normalized by the time scaling $\tau=\Omega t$ and is differentiated with respect to $\tau$. Prime $(.)^{\prime}$ represents a quantity differentiated with respect to $\tau$. Normalization in this way results in

$$
z^{\prime \prime}+\frac{2 \varepsilon \zeta \omega_{n}}{\Omega} z^{\prime}+\frac{\omega_{n}^{2}}{\Omega^{2}}(1+\varepsilon \delta \cos (\tau)) z+\frac{\omega_{n}^{2}}{\Omega^{2}}(\varepsilon \alpha+\varepsilon \gamma \cos (\tau)) z^{3}=0 .
$$

The parametric frequency $\Omega$ varies around the reference frequency $\Omega_{0}$ thus

$$
\Omega=\Omega_{0}(1-\varepsilon \Delta),
$$

Substituting Eq. (4) into Eq. (3) leads to a set of equations which are then simplified using the Taylor expansions $(1-\varepsilon \Delta)^{-1}=1+\varepsilon \Delta+\mathcal{O}\left(\varepsilon^{2}\right)$ and $(1-\varepsilon \Delta)^{-2}=1+2 \varepsilon \Delta+\mathcal{O}\left(\varepsilon^{2}\right)$, and ignoring high order terms.

If $\varepsilon=0$, the solution of $\mathrm{Eq}(3)$ are known, they are linear combination of $\cos (\tau)$ and $\sin (\tau)$. This linear combination can be written as

$$
z(\tau)=a \cos (\kappa \tau+\varphi),
$$

where $a$ and $\varphi$ are the constant amplitude and the phase which they can be determined from initial conditions. The frequency relation $\frac{\omega_{n}}{\Omega_{0}}=\kappa$ is used here for simplification.

If $\varepsilon \neq 0$ based on the method of Lagrange "variation of constant", we can assume that the solution can still be written in the above form but the amplitude and phase $a$ and $\varphi$ are now function of time. ${ }^{19}$ Hence, the complementary solution of the final simplified nonlinear equation (Eq. (3)) is a linear combination of $\cos (\Phi(\tau))$ and $\sin (\Phi(\tau))$, which can be written as 


$$
z(\tau)=a(\tau) \cos (\Phi(\tau))
$$

where $\Phi(\tau)=\kappa \tau+\varphi(\tau)$, and

$$
z(\tau)^{\prime}=a^{\prime}(\tau) \cos (\Phi(\tau))-a(\tau)\left(\kappa+\varphi^{\prime}(\tau)\right) \sin (\Phi(\tau))
$$

Substituting Eq. (6) and Eq. (7) into Eq. (3) results an equation which can be solved for $a^{\prime}(\tau)$ and $\varphi^{\prime}(\tau) . \bar{a}^{\prime}(\tau)$ and $\bar{\varphi}^{\prime}(\tau)$ are the averaged by assuming $a(\tau)$ and $\varphi(\tau)$ are changing slowly. $\bar{a}^{\prime}(\tau)$ and $\bar{\varphi}^{\prime}(\tau)$ are averaged over one period $T=\frac{2 \pi}{\Omega}$. The resulting averaged equation can be integrated respect to $\tau$ to find the $\bar{a}(\tau)$ and $\bar{\varphi}(\tau)$ for a given $\kappa$ and reference frequency $\Omega_{0}$. The steady-state behavior of the system can be recovered from the set of $\bar{a}^{\prime}(\tau)$ and $\bar{\varphi}^{\prime}(\tau)$ by setting $\left(\bar{a}^{\prime}, \bar{\varphi}^{\prime}\right)=(0,0)$ and solving for steady state values of $\bar{a}$ and $\bar{\varphi}$. The resulting solution is an approximation of the original solution. This method has been studied for the solution of the undamped and damped Mathieu equations. ${ }^{7,17}$

\subsection{First condition: $\kappa \neq \frac{1}{2}$ and $\Omega_{0} \neq 2 \omega_{n}$, damped response}

Solving the Eq. (3) with the method of averaging when $\kappa \neq \frac{1}{2}$ results in $a^{\prime}(\tau)$ and $\varphi^{\prime}(\tau)$ as follows

$$
\begin{gathered}
\bar{a}^{\prime}(\tau)=-\varepsilon \frac{\zeta \omega_{n}}{\Omega} \bar{a}, \\
\bar{\varphi}^{\prime}(\tau)=\kappa \varepsilon \Delta+\frac{3 \varepsilon \alpha \omega_{n}^{2}}{8 \kappa \Omega^{2}} \bar{a}^{2},
\end{gathered}
$$

Hence the solution based on the first approximation Eq. (5) is

$$
z(\tau)=\bar{a} \cos (\bar{\Phi})=\bar{a}_{0} \mathrm{e}^{-\varepsilon \frac{\zeta \omega_{n}}{\Omega}}
$$

$\bar{a}$ and $\bar{\Phi}$ are constant and they are replaced by $\bar{a}_{0}$ and $\bar{\Phi}_{0}$ to show they are based on the initial conditions.

In the time domain, where $\left(\kappa \tau=\omega_{n} t\right)$,

$$
z(t)=\bar{a}_{0} \mathrm{e}^{-\varepsilon \zeta \omega_{n} t}
$$

which is a decaying solution.

\subsection{Second condition: $\kappa=\frac{1}{2}$ and $\Omega_{0}=2 \omega_{n}$, steady-state response}

Solving the Eq. 3 with the method of averaging when $\kappa=\frac{1}{2}$ results in $a^{\prime}(\tau)$ and $\varphi^{\prime}(\tau)$ as follows

$$
\begin{gathered}
\bar{a}^{\prime}(\tau)=\bar{a}\left(\frac{\varepsilon \bar{a}^{2} \gamma}{8} \sin (2 \bar{\varphi})-\frac{\varepsilon \zeta \omega_{n}}{\Omega}+\frac{\varepsilon \delta}{8} \sin (2 \bar{\varphi})\right), \\
\bar{\varphi}^{\prime}(\tau)=\frac{1}{2} \varepsilon \Delta+\frac{\varepsilon \bar{a}^{2} \gamma}{8} \cos (2 \bar{\varphi})+\frac{3 \varepsilon \bar{a}^{2} \alpha}{16}+\frac{\varepsilon \delta}{8} \cos (2 \bar{\varphi}),
\end{gathered}
$$

where $\varepsilon \Delta=-\frac{\Omega}{2 \omega_{n}}+1$. The steady state solutions are given by $\bar{a}^{\prime}(\tau)=\bar{\varphi}^{\prime}(\tau)=0$. If $\bar{a}_{0}=0$ in Eq. (12) is zero, then $\bar{a}^{\prime}(\tau)=0$ but this means $z(\tau)=0$. Therefore, when $\bar{a}_{0}=0$ the system has trivial solutions, and has non-trivial solutions otherwise. 
2.3.1 Non-trivial solutions, $\bar{a}_{0} \neq 0$

When $\bar{a}_{0} \neq 0$, Eq. (12) becomes

$$
\frac{\varepsilon \bar{a}_{0}^{2} \gamma}{8} \sin (2 \bar{\varphi})-\frac{\varepsilon \zeta \omega_{n}}{\Omega}+\frac{\varepsilon \delta}{8} \sin (2 \bar{\varphi})=0
$$

and $\sin \left(2 \bar{\varphi}_{0}\right)$ and $\cos \left(2 \bar{\varphi}_{0}\right)$ are

$$
\begin{gathered}
\sin \left(2 \bar{\varphi}_{0}\right)=\frac{8 \zeta \omega_{n}}{\Omega\left(\bar{a}_{0}^{2} \gamma+\delta\right)} \\
\cos \left(2 \bar{\varphi}_{0}\right)= \pm\left(1-\left(\frac{8 \zeta \omega_{n}}{\Omega\left(\bar{a}_{0}^{2} \gamma+\delta\right)}\right)^{2}\right)
\end{gathered}
$$

When $\bar{\varphi}^{\prime}(\tau)=0$, from Eq. (13)

$$
\frac{1}{2} \varepsilon \Delta+\frac{\varepsilon \bar{a}_{0}^{2} \gamma}{8} \cos \left(2 \bar{\varphi}_{0}\right)+\frac{3 \varepsilon \bar{a}_{0}^{2} \alpha}{16}+\frac{\varepsilon \delta}{8} \cos \left(2 \bar{\varphi}_{0}\right)=0 .
$$

Substituting Eq. (16) into Eq. (17) yields the following relation for $\varepsilon \Delta$

$$
\varepsilon \Delta= \pm\left(1-\left(\frac{8 \zeta \omega_{n}}{\Omega\left(\bar{a}_{0}^{2} \gamma+\delta\right)}\right)^{2}\right)\left(\frac{\varepsilon \bar{a}_{0}^{2} \gamma}{4}+\frac{\varepsilon \delta}{4}\right)-\left(\frac{3 \varepsilon \alpha \bar{a}_{0}^{2}}{8}\right)
$$

where $\varepsilon \Delta=-\frac{\Omega}{2 \omega_{n}}+1$. This equation can be solved for $\bar{a}_{0}$ to be used for amplitude-frequency relation. If we solve this equation for $\bar{a}_{0}$, two solutions will be found.

\subsubsection{Stability of steady-state solutions}

The stability of a linear time-varying system, such as when $\alpha=0$ and $\gamma=0$ in Eq. (3), does not need to be determined with Lyapunov theorem stability criteria due to its relative simplicity. ${ }^{20,21}$ The solution of linear parametrically excited is stable if it is decaying in time and is unstable otherwise. ${ }^{20,21}$ However for a nonlinear system, such as the cubic-nonlinear system in Eq. (3) when $\alpha \neq 0$ and $\gamma=0$, we consider the small variation which can be added to the amplitude and the phase and then look at the state of the solutions. Hence, let $\bar{a}=\bar{a}_{0}+u$ and $\bar{\varphi}=\bar{\varphi}_{0}+v$, and substituting them into Eq. (12) and Eq. (13) results in set of equations which they can be linearized by ignoring higher terms of $u$ and $v$ and $u v$. After linearization the set of equations are

$$
\left\{\begin{array}{l}
u^{\prime} \\
v^{\prime}
\end{array}\right\}=\left[\begin{array}{cc}
0 & \varepsilon\left(\frac{\delta}{4} \bar{a}_{0}+\frac{\gamma}{4} \bar{a}_{0}^{3}\right) \cos \left(2 \bar{\varphi}_{0}\right) \\
\frac{3 \varepsilon \alpha \bar{a}_{0}}{8} & -\varepsilon\left(\frac{\delta}{4}+\frac{\gamma}{4} \bar{a}_{0}^{2}\right) \sin \left(2 \bar{\varphi}_{0}\right)
\end{array}\right]\left\{\begin{array}{l}
u \\
v
\end{array}\right\}
$$

the eigenvalues are

$$
\lambda_{1}, \lambda_{2}=-\frac{\varepsilon}{2}\left(\frac{\delta}{4}+\frac{\gamma}{4} \bar{a}_{0}^{2}\right) \sin \left(2 \bar{\varphi}_{0}\right) \pm \frac{1}{2} \sqrt{\left(\varepsilon\left(\frac{\delta}{4}+\frac{\gamma}{4} \bar{a}_{0}^{2}\right) \sin \left(2 \bar{\varphi}_{0}\right)\right)^{2}+\left(\frac{3 \varepsilon^{2} \alpha}{2}\left(\frac{\delta}{2} \bar{a}_{0}^{2}+\frac{\gamma}{4} \bar{a}_{0}^{4}\right)\right) \cos \left(2 \bar{\varphi}_{0}\right)}
$$

The steady state phase can be calculated from Eq. (15) and Eq. (16), and the solutions are $z(\tau)=\bar{a}_{0} \cos \left(\kappa \tau+\bar{\varphi}_{0}\right)$ when $\kappa=\frac{1}{2}$. In the case that two amplitudes, $\bar{a}_{01}$ and $\bar{a}_{02}$, nne of the solutions can be stable and the other one is the unstable solution. The stability of the parametrically excited system with nonlinear parameter Eq. (1) can be found using the eigenvalues in Eq. (20). Since $\left(\varepsilon\left(\frac{\delta}{4}+\frac{\gamma}{4} \bar{a}_{0}^{2}\right) \sin \left(2 \bar{\varphi}_{0}\right)\right)^{2}$ in Eq. (20) is always positive and negligible. Due to this, the following statements hold 
- If $\cos \left(2 \bar{\varphi}_{0}\right)>0$ then the eigenvalues can be positive real and negative real, hence an unstable solution exists.

- If $\cos \left(2 \bar{\varphi}_{0}\right)<0$ then eigenvalues are imaginary and negative real part, hence a stable solution exists.

- If $\cos \left(2 \bar{\varphi}_{0}\right)=0$ then the eigenvalues are negative and real, hence a stable solution exists.

The amplitude-frequency relations from Eq. (18) are found to be

$$
\begin{aligned}
& \bar{a}_{0,1}=\sqrt{\frac{-4 \varepsilon^{2} \gamma \delta-12 \varepsilon \alpha\left(\frac{\Omega}{\omega_{n}}\right)+24 \varepsilon \alpha-2\left(\frac{\omega_{n}}{\Omega}\right) \mathrm{j}_{1} \mathrm{j}_{2}}{4 \varepsilon^{2} \gamma^{2}-9 \varepsilon^{2} \alpha^{2}}}, \\
& \bar{a}_{0,2}=\sqrt{\frac{-4 \varepsilon^{2} \gamma \delta-12 \varepsilon \alpha\left(\frac{\Omega}{\omega_{n}}\right)+24 \varepsilon \alpha+2\left(\frac{\omega_{n}}{\Omega}\right) \mathrm{j}_{1} \mathrm{j}_{2}}{4 \varepsilon^{2} \gamma^{2}-9 \varepsilon^{2} \alpha^{2}}},
\end{aligned}
$$

where $\mathrm{j}_{1}$ and $\mathrm{j}_{2}$ are

$$
\begin{gathered}
\mathrm{j}_{1}=\sqrt{9 \varepsilon^{4}\left(\frac{\omega_{n}}{\Omega}\right)^{2} \alpha^{2} \delta^{2}+24 \varepsilon^{3} \gamma\left(\frac{\omega_{n}}{\Omega}\right)^{3} \alpha \delta+16 \varepsilon^{2} \gamma^{2}\left(\frac{\omega_{n}}{\Omega}\right)^{4}}, \\
\mathrm{j}_{2}=\sqrt{256 \varepsilon^{4} \gamma^{2} \zeta^{2}-48 \varepsilon^{3} \gamma\left(\frac{\omega_{n}}{\Omega}\right)^{2} \alpha \delta-576 \varepsilon^{4} \alpha^{2} \zeta^{2}-64 \varepsilon^{2} \gamma^{2}\left(\frac{\omega_{n}}{\Omega}\right)^{3}+64 \varepsilon^{2} \gamma^{2}\left(\frac{\omega_{n}}{\Omega}\right)^{2}} .
\end{gathered}
$$

Based on the stability of the non-trivial solutions, the solutions are

- $z(t)_{1}=\bar{a}_{0,1} \cos \left(\omega_{n} t+\bar{\varphi}_{0}\right)$ when $\bar{\varphi}_{0}>0$ or $\bar{\varphi}_{0}=0$, which is stable.

- $z(t)_{2}=\bar{a}_{0,2} \cos \left(\omega_{n} t+\bar{\varphi}_{0}\right)$ when $\bar{\varphi}_{0}<0$, which is unstable.

\subsection{Stability and transition curve}

With regards to the linear time-varying system for a given value of normalized parametric stiffness $\delta$ and the frequency ratio $\frac{\Omega}{\omega_{n}}$, there are bounded and unbounded solutions. If the solution is bounded then the corresponding point in the $\delta$ and $\frac{\Omega}{\omega_{n}}$ parameter space is stable. ${ }^{22}$ If the solution is unbounded (It can be exponentially growing), then the corresponding point in $\varepsilon \delta$ and $\frac{\Omega}{\omega_{n}}$ parameter plane is unstable. Unstable solutions occur when the parametric frequency is near and at the natural frequency $\left(\Omega \approx \omega_{n}\right)$, near and at twice the natural frequency $\left(\Omega \approx 2 \omega_{n}\right)$, and at smaller ratios of the natural frequency. For the nonlinear parametrically excited system steady-state solutions exist. Trivial and non-trivial solutions of a nonlinear system can be stable or unstable as it explained in Section 2.3.2. Normalized parametric stiffness $\delta$ and frequency ratio $\frac{\Omega}{\omega_{n}}$ relation can be found when the system has non-trivial solution by substituting $\varepsilon \Delta=1-\frac{\Omega}{2 \omega_{n}}$ into Eq. (18), and solving for $\varepsilon \delta$ as follows

$$
\varepsilon \delta=-\varepsilon \bar{a}_{0}{ }^{2} \gamma+\frac{1}{2} \sqrt{9 \varepsilon \alpha^{2} \bar{a}_{0}{ }^{4}-48 \varepsilon \bar{a}_{0}{ }^{2} \alpha\left(\frac{\Omega}{2 \omega_{n}}-1\right)+64\left(\frac{\Omega}{2 \omega_{n}}-1\right)^{2}+16 \varepsilon \zeta^{2}} .
$$

This equation shows where the system in Eq. (2) has steady-state solutions. This equation is called the transition curve for the nonlinear parametric system. Although the transition curve shows where the system has steadystate solutions, it is not sufficient to identify where the solution is unstable. In order to find out where in the transition curve the unstable solutions exist, we should map the amplitude-frequency response plot along with the transition curve plot. 
From Eq. (21) and Eq. (22), the amplitude-frequency relation is plotted in Fig. 1. The lower plot in Fig. 1 is the analytical amplitude-frequency response of Eq. (2) when $\zeta=0.0316, \omega_{n}=31.62 \mathrm{Rads}^{-1}, \varepsilon=1, \alpha=150 \mathrm{~m}^{-2}$ and $\gamma=80 \mathrm{~m}^{-2}$. The stable non-trivial solutions (the upper line) were also found numerically using ODE45. The lower line (dash-dot) shows the unstable solutions that could not be found numerically. The cubic nonlinearity in the system is a hardening nonlinearity, hence the curve is shifted towards higher frequencies. ${ }^{23}$

When $\bar{a}_{0}=0$, the effect of nonlinearity will be zero as the nonlinear terms are amplitude dependent and the system can be treated as a linear time-varying system. However $\bar{a}_{0}=0$ does not mean that the system cannot be unstable; it will become unstable as a result of the parametric term in Eq. (2).

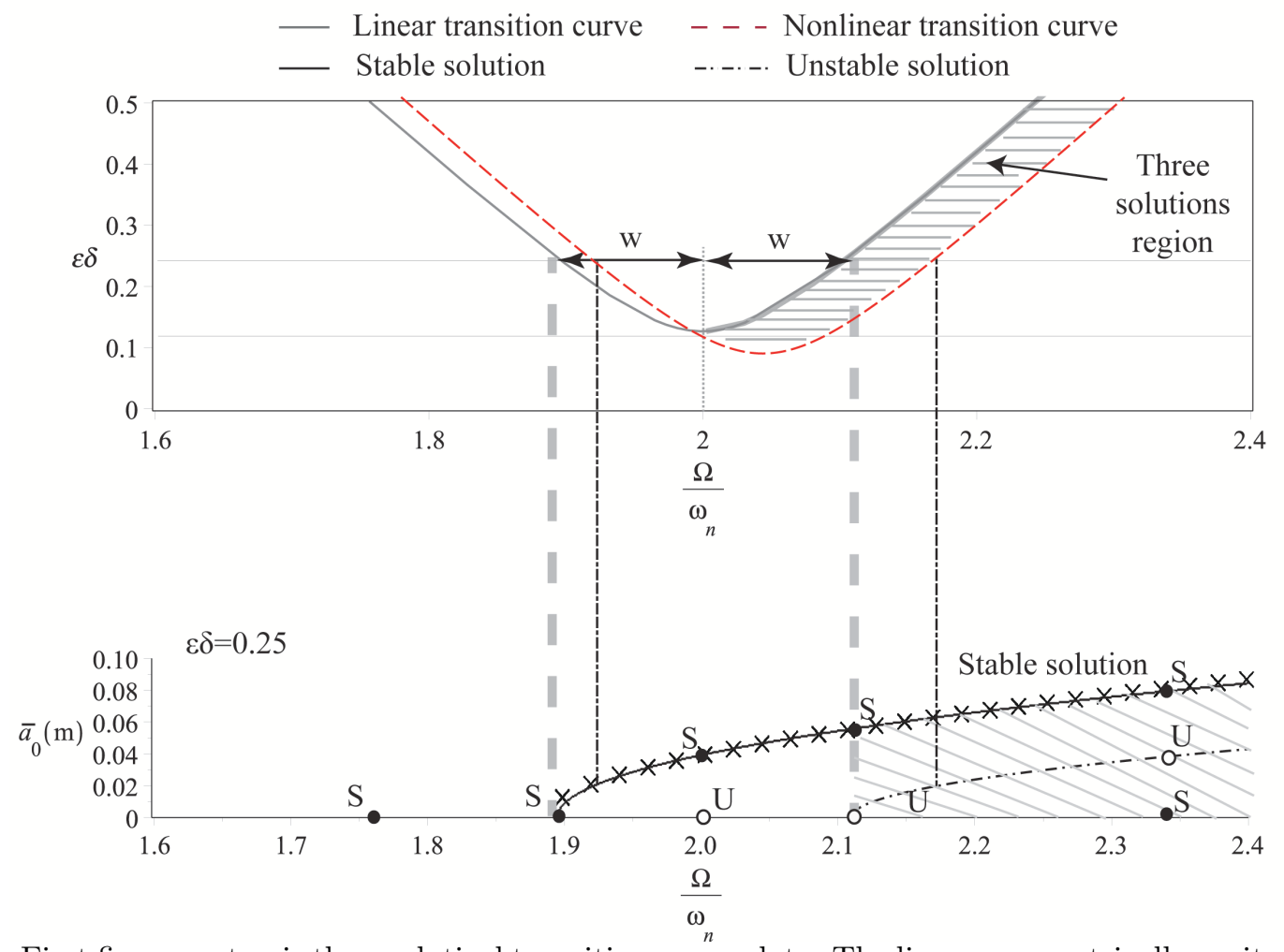

Figure 1. First figure on top is the analytical transition curve plots. The linear parametrically excited transition curve (Gray solid line) and the nonlinear parametrically excited transition curve (Dashed black line) is plotted based on Eq. (25) for $\bar{a}_{0}=0.02 \mathrm{~m}$. The shaded area in nonlinear transition curve shows there exist three solution, trivial stable, non-trivial stable and non-trivial unstable. Inside the nonlinear transition curve steady-state nontrivial solutions exist. The second figure is the amplitude-frequency plot for the nonlinear parametrically excited system for $\varepsilon \delta=0.25$. The steady-state solutions are found analytically as well as numerically (cross points). The analytical and numerical results are in agreement.

For the case $\bar{a}_{0}=0$, the transition curve from Eq. (25) can be calculated and plotted. The solid grey transition curve in Fig. 1 (top) shows the transition curve for a linear parametrically excited system when $\bar{a}_{0}=0$. For the linear parametrically excited system, unbounded unstable solutions exist inside the transition curve, and bounded solutions exist outside. At $\varepsilon \delta=0.25$ on the transition curve, the system is unstable when the frequency ratio is $1.9 \leq \frac{\Omega}{\omega_{n}} \leq 2.1$. From the amplitude-frequency relation in Fig. 1 it can be seen that unstable solutions exist when $\bar{a}_{0}=0$ and $1.9 \leq \frac{\Omega}{\omega_{n}} \leq 2.1$. Two lines from the transition curve to the amplitude-frequency plot shows the area in which the unstable trivial solutions exist. Instability of a linear parametrically excited systems has been discussed in other work. ${ }^{22,24}$

When $\bar{a}_{0} \neq 0$ and non-trivial solutions exist, the corresponding transition curve can be found from Eq. (25). The transition curve is also dependent on $\bar{a}_{0}$; the nonlinear transition curve for $\bar{a}_{0}=0.02 \mathrm{~m}$ is plotted as the top 
dashed black curve in Fig. 1. The transition curve is shifted to the right relative to the linear transition curve as a result of the hardening nonlinearity, and is also shifted down as a result of the cubic parametric nonlinearity. This change is explained and measured experimentally in Section 3.

At $\varepsilon \delta=0.25$ value on the transition curve, the nonlinear system has a steady-state response for the frequency ratio $1.9 \leq \frac{\Omega}{\omega_{n}} \leq 2.1$. The two vertical lines from the transition curve to the amplitude-frequency plot shows the corresponding amplitude-frequency plot. From the amplitude-frequency plot, stable and unstable solutions can be found when $2.1 \leq \frac{\Omega}{\omega_{n}} \leq 2.17$. Hence in the transition curve there is an area in which the system has stable and unstable solutions. The transition curve at $\varepsilon \delta=0.25$ and $1.93 \leq \frac{\Omega}{\omega_{n}} \leq 2.1$ has only one trivial solution which is stable. For $2.1 \leq \frac{\Omega}{\omega_{n}} \leq 2.17$ in the transition curve, both stable and unstable solutions exist. The area in the transition curve for a nonlinear system where non-trivial solutions exist are shaded in grey. This area can also be found analytically based on the difference between transition curves for the linear and nonlinear systems. The width (w) for a linear transition curve can be found as a function of excitation frequency from Eq. (25) when $\bar{a}_{0}=0$. The phase-portrait plot for some points in transition curve would explain the behavior of the solutions, this will be considered for looking at the solutions in details. ${ }^{25}$

The relation between the amplitude-frequency plot and the transition curve clarifies the difference between the transition curve for a linear parametrically excited and a nonlinear parametrically excited system. This will enable the experimental transition curve for a nonlinear system to be plotted based on the characteristic of the nonlinear system. Experimentally speaking, only the non-trivial stable solutions can be captured.

\section{EXPERIMENTAL RESULTS}

In order to validate the analytical model outlined in the previous sections, it is necessary to devise an experimental model from which average dissipated powers can be obtained. The performance of this experimental model will determine the feasibility of the analytical model for designing a piezoelectric vibration energy harvester. This model consists of an electromagnetic system, which emulates the stiffness of the parametrically excited system. A cantilever beam acts as a harvester which can be excited from the base from the structure. Fig. 2(a) shows the experimental set-up used to generate the periodic electromagnetic stiffness on a cantilever beam. A signal generator and a LAMBDA Zup 10-20 amplifier were used to generate DC and AC current through the coils. A QUATTRO Data Physics DAQ and NI DAQ systems were used to record data. The beam displacement was measured by a POLYTECH OFV056 laser vibrometer. An aluminium support was used to clamp the cantilever beam in a fixed orientation and location. In order to decrease the vertical movement of the beam, the beam has to be precisely located between the axis of the two coils. This position was adjusted by entering the attractive mode of the magnets, and returning to the repulsion mode once the centered state is reached. The data in this paper was recorded when the electromagnetic system was $0.34 \mathrm{~m}$ away from the support end. The vibrometer reads the velocity of the beam at the tip. The electromagnetic system and the beam configuration are provided in Table 1.

An electromagnetic system is used to emulate the time-variant stiffness in the experimental model. The electromagnetic device consists of a pair of magnets and a pair of coils used to alter the stiffness of the cantilever beam as shown in Fig. 2(a). The pair of magnets are fixed to the beam and the pair of coils is fixed to a wood support. The coils have the same number of turns and winding direction. In order to emulate the extra stiffness in the beam, the mutual acting forces between the coils and magnets cause the electromagnetic device to act like a spring 2(b). If the magnets and coils are repulsive, the magnets and the attached beam return to their equilibrium position when the beam is displaced. In this case the stiffness produced by the electromagnetic device is positive. If the magnets are reversed, an attractive force is generated and negative stiffness is obtained. The negative stiffness comes from the fact that the attractive force pushes the magnet away from equilibrium position. The time-varying stiffness is proportional to the current through the coil. The electromagnetic system, the magnets and coils are shown in Fig. 2(b). The magnetic field produced by the coils and the magnets can be calculated assuming that the coils are perfect and identical solenoids and that the magnets constitute a magnetic dipole. In Fig. 2(c) the cantilever beam between the two magnets are considered to be at equilibrium when the two coils are traversed by the same current, the magnets are positioned at the same distance $h$ from the dipole, 


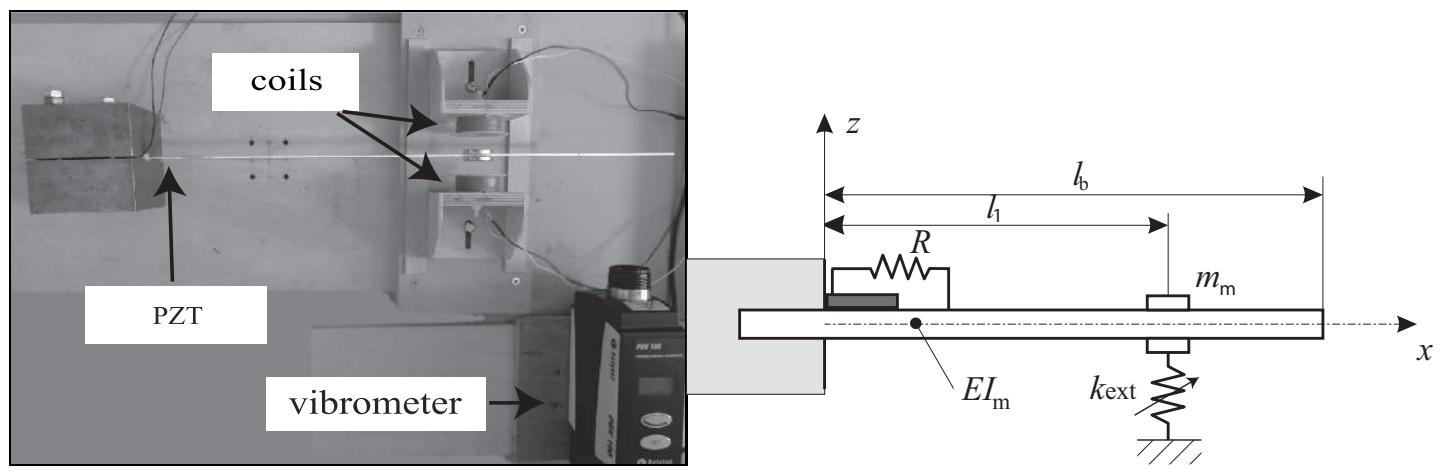

(a)

(b)

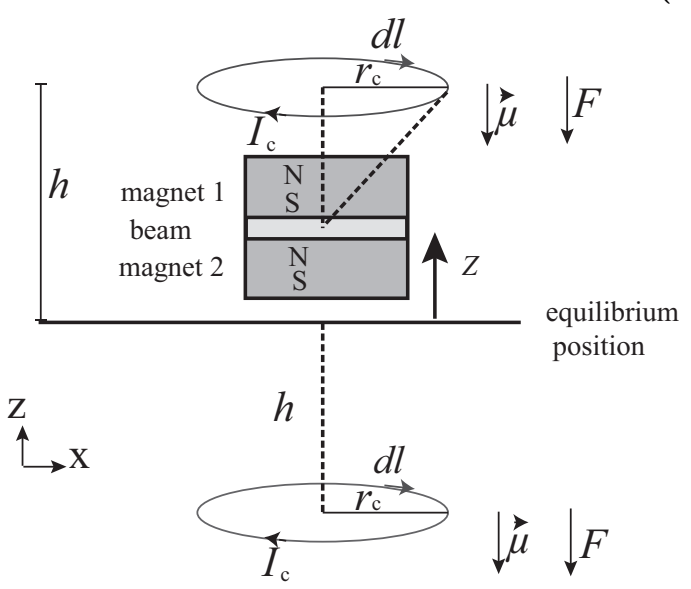

(c)

Figure 2. (a) A cantilever beam with an electromagnetic part consists of two coils and magnets and a piezoelectric patch close to the fixed part. (b) Schematic of the cantilever beam model with time-varying stiffness. (c) Schematic of the coils and the magnets in repulsion.

and the magnetic field produced by the coils causes no movement along the $z$ axis. The repulsive magnetic force $F_{e}$ is $^{26}$

$$
F_{e}(z)=-\frac{3 \mu \mu_{0} r_{c} I_{c} N(h+z)}{2\left((h+z)^{2}+r_{c}^{2}\right)^{5 / 2}}-\frac{3 \mu \mu_{0} r_{c} I_{c} N(z-h)}{2\left((z-h)^{2}+r_{c}^{2}\right)^{5 / 2}} .
$$

where $\mu$ is the magnetic dipole moment, $N$ is the identical coils number of turns, $\mu_{0}$ is the permeability of free space, $r_{c}$ is the coil radius, and $h$ is the half distance between coils. Other parameters are specified in table 1. Using the Taylor expansion about $z=0$, Eq. (26) can be expressed as

$$
F_{e}(z)=\mathrm{H}_{1} I_{c} z+\mathrm{H}_{2} I_{c} z^{3},
$$

where $\mathrm{H}_{1}$ and $\mathrm{H}_{2}$ are

$$
\mathrm{H}_{1}=\left(\frac{-2 G}{\left(h^{2}+r_{c}^{2}\right)^{\frac{5}{2}}}+\frac{10 G h^{2}}{\left(h^{2}+r_{c}^{2}\right)^{\frac{7}{2}}}\right) \text {, and } \mathrm{H}_{2}=\left(\frac{5 G}{\left(r_{\mathrm{c}}^{2}+h^{2}\right)^{\frac{7}{2}}}-\frac{2.8 G h^{2}}{\left(r_{\mathrm{c}}^{2}+h^{2}\right)^{\frac{9}{2}}}+\frac{4.2 G h^{4}}{\left(r_{\mathrm{c}}^{2}+h^{2}\right)^{\frac{11}{2}}}\right),
$$

where $G$ is

$$
G=\frac{1}{2} \mu \mu_{0} r_{c} N
$$


Table 1: Mechanical properties and dimensions

\begin{tabular}{lll}
\hline Property & Value & Units \\
\hline Material of the cantilever beam & Aluminium & \\
Young's Modulus of the beam $(E)$ & 70 & $\mathrm{GPa}$ \\
Beam mass $\left(m_{\mathrm{b}}\right)$ & 0.034 & $\mathrm{~kg}$ \\
Constant stiffness of the beam $\left(k_{b}\right)$ & 42.7 & $\mathrm{Nm}^{-1}$ \\
Beam length $\left(l_{\mathrm{b}}\right)$ & 0.54 & $\mathrm{~m}$ \\
Beam width $\left(b_{\mathrm{b}}\right)$ & 0.01 & $\mathrm{~m}$ \\
Beam thickness $\left(t_{\mathrm{b}}\right)$ & 0.002 & $\mathrm{~m}$ \\
\hline Magnets mass $\left(m_{\mathrm{b}}\right)$ & 0.035 & $\mathrm{~kg}$ \\
Magnets outer radius $\left(r_{\mathrm{c}}\right)$ & 0.015 & $\mathrm{~m}$ \\
Magnets inner radius & 0.0025 & $\mathrm{~m}$ \\
Magnets thickness & 0.005 & $\mathrm{~m}$ \\
Magnets mounting position $\left(l_{1}\right)$ & 0.34 & $\mathrm{~m}$ \\
Permanent coils outer radius & 0.022 & $\mathrm{~m}$ \\
Permanent coils inner radius & 0.009 & $\mathrm{~m}$ \\
Half distance between the coils $(h)$ & 0.035 & $\mathrm{~m}$ \\
Number of coil turns $(N)$ & 754 & $(\mathrm{Unitless})$ \\
\hline Piezoelectric length & 0.003 & $\mathrm{~m}$ \\
Piezoelectric width & 0.01 & $\mathrm{~m}$ \\
Piezoelectric thickness & 0.0005 & $\mathrm{~m}$ \\
Piezoelectric capacitance & 8.3 & $\mathrm{nF}$ \\
Effective piezoelectric coefficient & $-2.39 \times 10^{-5}$ & $\mathrm{NV}$ \\
Shunted optimal load resistance $\left(R_{\mathrm{opt}}\right)$ & 1 & $\mathrm{MOhm}$ \\
\hline
\end{tabular}

Considering the interaction of the dynamics of the beam with the electromagnetic system, the simplified single degree of freedom system motion equation is same as Eq. (1) where parameters $k_{1}, k_{\mathrm{p} 1}, k_{3}$ and $k_{\mathrm{p} 3}$ as a function of currents are $k_{1}=k_{b}+k_{\mathrm{ext}}, k_{\mathrm{ext}}=\mathrm{H}_{1} I_{\mathrm{DC}}, k_{\mathrm{p} 1}=\mathrm{H}_{1} I_{\mathrm{AC}}, k_{3}=\mathrm{H}_{2} I_{\mathrm{DC}}$ and $k_{\mathrm{p} 3}=\mathrm{H}_{2} I_{\mathrm{AC}},{ }^{27}$ where $k_{b}$ is the beam stiffness independent of the electromagnetic system. $I_{\mathrm{DC}}$ and $I_{\mathrm{AC}}$ are the $\mathrm{DC}$ current and $\mathrm{AC}$ current flow in the coils. The current in the coil is $I_{\mathrm{c}}=I_{\mathrm{DC}}+I_{\mathrm{AC}} \cos (\Omega t)$ with frequency of $\Omega$. For simplicity, expressions involving the quantities $k_{1}, k_{\mathrm{p}}, k_{3}$, and $k_{\mathrm{p} 3}$ will not explicitly state that they relate to current. During the experimental tests, the electromagnetic system was positioned in repulsion mode, which resulted in a positive stiffness in the cantilever beam. Impact testing was carried out to determine the natural frequency for different DC currents. An impulsive excitation was applied to the cantilever beam by an instrumented hammer. The displacement at the tip of the beam was measured using a laser vibrometer.

The first natural frequency of the cantilever beam with the electromagnetic system can be found using the Rayleigh Energy Method as ${ }^{28}$

$$
\omega_{n}=\left(\frac{61.4 E I_{\mathrm{m}}+\left(k_{\mathrm{ext}}\right) D_{1}}{0.52 m_{\mathrm{b}}+m_{\mathrm{m}} D_{1}}\right)^{\frac{1}{2}}
$$

where

$$
D_{1}=-0.68 \cos \left(\frac{2 \pi l_{1}}{\lambda}\right)+0.68 \cosh \left(\frac{2 \pi l_{1}}{\lambda}\right)+0.5 \sin \left(\frac{2 \pi l_{1}}{\lambda}\right)-0.5 \sinh \left(\frac{2 \pi l_{1}}{\lambda}\right),
$$

and where $m_{\mathrm{b}}$ is the mass of the beam, $m_{\mathrm{m}}$ is the combined mass of the magnets, $E$ is the Young's modulus, $I_{\mathrm{m}}$ is the moment of inertia, $l_{1}$ is the distance of the magnets and the coils with respect to the fixing point, and $\lambda$ is the wavenumber. Fig. 2 (c) illustrates some of these properties. The relationship between electromagnetic stiffness and the current flow in the coils found analytically is implemented here. From the relation between the natural frequency and electromagnetic stiffness, the current flow can be obtained in terms of the natural frequency by substituting $k_{\text {ext }}$ into Eq. (30). The amplitude of both parametric, cubic and cubic parametric stiffness, are function of the distance between the coils $(h)$. The distance $h=0.035 \mathrm{~m}$ is chosen to check the stability of the system because at this point the system is under positive cubic and cubic nonlinearity. 


\subsection{Transition curve for a parametrically excited beam with cubic and cubic parametric nonlinearity}

The experimental test for finding the transition curve for a system with $\zeta=0.037, \omega_{n}=34.28 \mathrm{Rads}^{-1}, \varepsilon=1$, and $\alpha=195.7 \mathrm{~m}^{-2}$ when $I_{\mathrm{DC}}=4.2 \mathrm{~A}$ and $I_{\mathrm{AC}}$ is changing at each point in the transition curve to generate different parametric stiffness $\delta$ and as a result different cubic parametric stiffness $\gamma$, were considered. Both $\delta$ and $\gamma$ were function of AC current.

The analytical transition curve for a given experimental parameters $\zeta=0.037, \omega_{n}=34.28 \mathrm{Rad} \mathrm{s}^{-1}, \varepsilon=1$, $\alpha=195.7 \mathrm{~m}^{-2}$ and amplitude $\bar{a}_{0}=0.01 \mathrm{~m}$ based on Eq. (25) is plotted for the nonlinear system in Fig. 3a, and when $\bar{a}_{0}=0$, it is plotted for a linear parametrically excited system. It is expected that the transition curve for the nonlinear system shifts to the right as a result of cubic nonlinearity and shifts down as a result of cubic parametric nonlinearity. This shift down decreases the instability threshold which have been a problem in making the harvester parametric in previous introduced systems in literature. ${ }^{25,29}$ Hence applying the cubic parametric nonlinearity is beneficial for the parametric harvester to have a steady-state response with a lower parametric amplitude. Parametric amplification has been studied for different applications in macro scale devices. ${ }^{14,30,31}$ For the system introduced here, the parametric amplitude $\delta$ is a function of $\mathrm{AC}$ current and the sooner the system becomes parametric and reaches the instability threshold, the lower the power consumed by the parametrically excited energy harvester. The experimental results for the transition curve when the stable responses are captured shows the system has potential to generate the steady-state responses inside the transition curve plotted in Fig. $3 \mathrm{~b}$ as long as the system is excited by an initial amplitude $\bar{a}_{0}=0.01 \mathrm{~m}$.

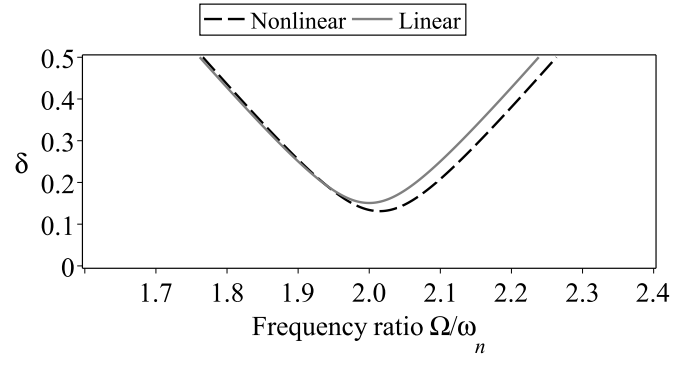

(a)

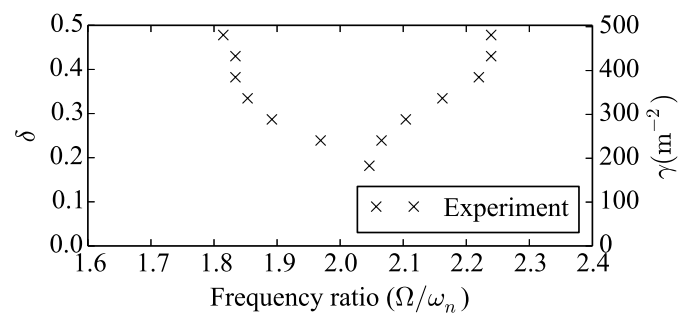

(b)

Figure 3. (a) The analytical transition curve for a given experimental parameters $\zeta=0.037, \omega_{n}=34.28 \mathrm{Rads}^{-1}$, $\varepsilon=1, \alpha=195.7 \mathrm{~m}^{-} 2, \gamma=195.7 \mathrm{~m}^{-2}$ and amplitude $\bar{a}_{0}=0.01 \mathrm{~m}$ based on Eq. (25) for the nonlinear system and for a linear system when $\bar{a}_{0}=0$.(b) The experimental transition curve when the system has positive cubic and cubic parametric nonlinearity. The cubic parametric nonlinearity changes as the AC current changes for each point in transition curve, which this is not included in the analytical transition curve. The analytical transition curve is plotted for a fixed cubic parametric nonlinearity which is the first point in experimental transition curve $\gamma=195.7 \mathrm{~m}^{-2}$.

\section{EXPERIMENTAL AVERAGE DISSIPATED POWER AND BROADENING THE FREQUENCY BANDWIDTH}

A piezoelectric element is attached to the beam near the fixed end. The element is a PZT ceramic PIC255 model, from Physik Instrumente (PI). An optimum resistive load $R_{\text {opt }}$ is shunted to the piezoelectric element in order to estimate the power that can be delivered to an electric load. ${ }^{32}$ The voltage across the resistive load is used to find the dissipated power. The average dissipated power from the load resistance for one cycle $T=\frac{2 \pi}{\Omega}$ is

$$
P_{\mathrm{av}}=\frac{1}{T} \int_{0}^{T} \frac{v(t)^{2}}{R_{\mathrm{opt}}} \mathrm{d} t
$$

Four different experimental tests were carried out to measure the voltage and the average power across the resistor. The specification of each test is shown in Table 2. These four tests are considered for two different 


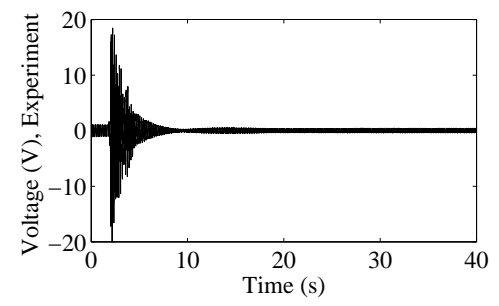

(a)

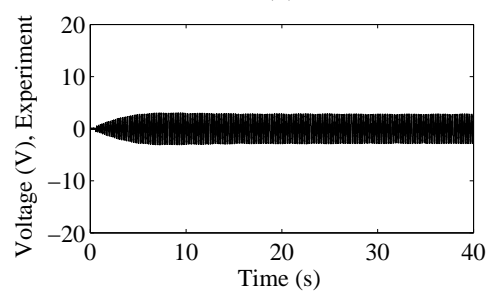

(c)

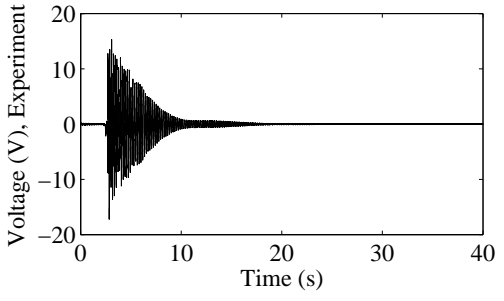

(b)

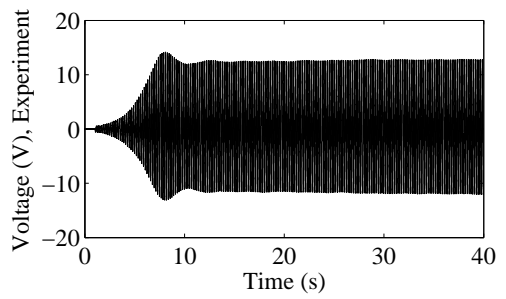

(d)

Figure 4. (a) The measured voltage across the optimum load for a transient response when $\Omega=\omega_{n}$ and $\delta=0.08$. (b) $\Omega=2 \omega_{n}$ and $\delta=0.08$. (c) The measured velocity for a steady-state response when $\Omega=\omega_{n}$ and $\delta=0.43$. (d) A steady-state response when $\Omega=2 \omega_{n}$ and $\delta=0.25$.

transient and steady-state cases. The parametric stiffnesses and parametric frequencies in Table 2 are chosen based on the experimental transition curve in Fig. 3b.

Table 2: Average dissipated power

\begin{tabular}{lllll}
\hline Free response tests & $\delta$ & $\Omega$ & $P_{\mathrm{av}}$ (Theory) & $P_{\mathrm{av}}$ (Measured) \\
\hline Transient (Fig. 4(a)) & 0.08 & $\omega_{n}$ & $0.4 \mu \mathrm{W}$ & $0.31 \mu \mathrm{W}$ \\
Transient (Fig. 4(b)) & 0.08 & $2 \omega_{n}$ & $0.44 \mu \mathrm{W}$ & $0.53 \mu \mathrm{W}$ \\
Steady-state (Fig. 4(c)) & 0.43 & $\omega_{n}$ & $0.6 \mu \mathrm{W}$ & $0.57 \mu \mathrm{W}$ \\
Steady-state (Fig. 4(d)) & 0.25 & $2 \omega_{n}$ & $8.7 \mu \mathrm{W}$ & $7.8 \mu \mathrm{W}$
\end{tabular}

Upon comparison, the theoretical and experimental average absorbed powers are in moderate agreement. The comparison between the two steady-state cases at the natural frequency and at twice the natural frequency shows that more power can be harvested in the case where $\Omega=2 \omega_{n}$ compared with when $\Omega=\omega_{n}$ in both the experimental and analytical cases; this is due to the increased steady-state velocity. This effect results in a factor 14.5 increase in the theoretical case, and a factor 13.6 increase in the experimental case. In the transient case, an increase in dissipated power is also observed, but this result primarily shows that a parametrically excited harvester is better tuned for steady-state response.

As a result of the cubic and cubic parametric nonlinearity, the amount of power that can be harvested with the parametric harvester is not only high at the natural frequencies but is also high at other frequencies close to twice the natural frequency as inside the transition curve only steady-state responses were found. However the parametric harvester itself needs power to generate the parametric stiffness as well as the nonlinear terms which this could be improved by changing the design of the electromagnetic system to use less power and generate more. The efficiency of the electromagnetic system could be improved by increasing the number of turns in the coils and also having an magnetic field flow around the experimental setup. This will be considered as a future work.

\section{SUMMARY AND CONCLUSIONS}

This paper investigated vibration energy harvesting from a nonlinear parametrically excited harvester. It has been shown that such systems have large amplitudes of vibrations due to their complex dynamics, that can be used for energy harvesting. An analytical model for a nonlinear parametrically excited harvester based on the method of averaging was explained. An amplitude-frequency plot was provided, showing the regions in which 
the dynamics of the model are stable or unstable. The responses of the system for transient and steady-state were obtained. The transition curve for the nonlinear system and its relation with the amplitude-frequency were clarified.

An experimental model of the parametrically excited vibration energy harvester was proposed, which used electromagnetic coils and magnets to emulate time-varying stiffness, and a cantilever beam as a structure. Stiffness of the system was expressed as a function of current passed through the coils of the electromagnet, and simplification of the nonlinearity term relating stiffness to current was justified. Natural frequency was then related to the stiffness, which in turn created a relationship between natural frequency and current. The analytical results were validated using experiments. The resulting experimental model was used to determine the transition curves.

In conclusion, the experimental model validated the analytical model. The transition curves obtained both analytically and experimentally were in reasonable agreement, though there were some discrepancies due to model simplifications. The peak power dissipated by the experimental model was also comparable to the equivalent analytical result, and the distribution of powers demonstrated that a harvester designed by these principles will have a degree of robustness; a slight change in the frequency of excitation will still result in some power being harvested. Ultimately, a framework has been established in which parametrically excited systems could be exploited in the design of vibration energy harvesters.

\section{ACKNOWLEDGMENTS}

The authors would like to thank Stefan Neumeyer from Thechnical University of Denmark to contribute in understanding the nonlinear parametrically excited systems.

\section{REFERENCES}

1. M. F. Daqaq, R. Masana, A. Erturk, and D. D. Quinn, "On the role of nonlinearities in vibratory energy harvesting: A critical review and discussion," Applied Mechanics Reviews 66(4), p. 040801, 2014.

2. S. P. Beeby, M. J. Tudor, and N. M. White, "Energy harvesting vibration sources for microsystems applications," Measurement Science and Technology 17, pp. R175-R195, Dec. 2006.

3. D. Zhu, M. J. Tudor, and S. P. Beeby, "Strategies for increasing the operating frequency range of vibration energy harvesters: a review," Measurement Science and Technology 21(2), p. 022001, 2010.

4. L. Tang, Y. Yang, and C. K. Soh, "Toward broadband vibration-based energy harvesting," Journal of Intelligent Material Systems and Structures 21(18), pp. 1867-1897, 2010.

5. A. Cammarano, S. A. Neild, S. G. Burrow, and D. J. Inman, "The bandwidth of optimized nonlinear vibration-based energy harvesters," Smart Materials and Structures 23(5), p. 055019, 2014.

6. S.-J. Jang, E. Rustighi, M. J. Brennan, Y. P. Lee, and H.-J. Jung, "Design of a 2dof vibrational energy harvesting device," Journal of Intelligent Material Systems and Structures 22(5), pp. 443-448, 2011.

7. W.-C. Xie, Dynamic stability of structures, Cambridge University Press, 2006.

8. A. P. da Costa, J. Martins, F. Branco, and J.-L. Lilien, "Oscillations of bridge stay cables induced by periodic motions of deck and/or towers," Journal of Engineering Mechanics 122(7), pp. 613-622, 1996.

9. M. F. Daqaq, C. Stabler, Y. Qaroush, and T. Seuaciuc-Osorio, "Investigation of Power Harvesting via Parametric Excitations," Journal of Intelligent Material Systems and Structures 20, pp. 545-557, Nov. 2008.

10. M. F. Daqaq and D. Bode, "Exploring the parametric amplification phenomenon for energy harvesting," Proceedings of the Institution of Mechanical Engineers, Part I: Journal of Systems and Control Engineering 225, pp. 456-466, June 2011.

11. Y. Jia, J. Yan, K. Soga, and A. A. Seshia, "A parametrically excited vibration energy harvester," Journal of Intelligent Material Systems and Structures 25, pp. 278-289, June 2013.

12. K. Kecik and M. Borowiec, "An autoparametric energy harvester," The European Physical Journal Special Topics 222(7), pp. 1597-1605, 2013. 
13. Y. Jia, J. Yan, K. Soga, and A. A. Seshia, "Parametrically excited MEMS vibration energy harvesters with design approaches to overcome the initiation threshold amplitude," Journal of Micromechanics and Microengineering 23, p. 114007, Nov. 2013.

14. J. F. Rhoads, N. J. Miller, S. W. Shaw, and B. F. Feeny, "Mechanical domain parametric amplification," Journal of Vibration and Acoustics 130(6), p. 061006, 2008.

15. J. F. Rhoads and S. W. Shaw, "The impact of nonlinearity on degenerate parametric amplifiers," Applied Physics Letters 96(23), p. 234101, 2010.

16. G. Prakash, A. Raman, J. Rhoads, and R. G. Reifenberger, "Parametric noise squeezing and parametric resonance of microcantilevers in air and liquid environments," Review of Scientific Instruments 83(6), p. 065109, 2012.

17. J. A. Sanders, F. Verhulst, and J. Murdock, Averaging methods in nonlinear dynamical systems, vol. 59, Springer, 2007.

18. F. Dohnal and F. Verhulst, "Averaging in vibration suppression by parametric stiffness excitation," Nonlinear Dynamics 54(3), pp. 231-248, 2008.

19. F. Verhulst, Nonlinear differential equations and dynamical systems, Springer Science \& Business Media, 1996.

20. H. K. Khalil and J. Grizzle, Nonlinear systems, vol. 3, Prentice hall New Jersey, 1996.

21. S. Sastry, Nonlinear systems: analysis, stability, and control, vol. 10, Springer New York, 1999.

22. A. H. Nayfeh and D. T. Mook, Nonlinear oscillations, John Wiley \& Sons, 2008.

23. M. F. Daqaq, "Role of stiffness nonlinearities in the transduction of energy harvesters under white gaussian excitations," in Advances in Energy Harvesting Methods, pp. 163-190, Springer, 2013.

24. B. Zaghari, E. Rustighi, and M. Ghandchi Tehrani, "Experimental study on harvesting energy from a parametrically excited system," in MoViC2014: The 12th International Conference on Motion and Vibration Control, August 2014.

25. L. Ng and R. Rand, "Bifurcations in a mathieu equation with cubic nonlinearities," Chaos, Solitons 83 Fractals 14(2), pp. 173-181, 2002.

26. D. C. Jolly, "Identity of the ampere and biot-savart electromagnetic force laws," Physics letters A 107(5), pp. 231-234, 1985.

27. B. Zaghari, M. Ghandchi Tehrani, and E. Rustighi, "Mechanical modelling of a vibration energy harvester with time-varying stiffness," in EURODYN 2014: 9th International Conference on Structural Dynamics, 2014.

28. W. Thomson, Theory of vibration with applications, CRC Press, 1996.

29. L. Cveticanin and I. Kovacic, "Parametrically excited vibrations of an oscillator with strong cubic negative nonlinearity," Journal of Sound and Vibration 304(1), pp. 201-212, 2007.

30. S. Neumeyer and J.-J. Thomsen, "Jumps and bi-stability in the phase-gain characteristics of a nonlinear parametric amplifier," in 8th European Nonlinear Dynamics Conference,

31. S. Neumeyer and J. J. Thomsen, "Macroscale mechanical domain parametric amplification: superthreshold pumping and optimal excitation parameters," in EUROMECH Colloquium 532, 2012.

32. S. Roundy and Y. Zhang, "Toward self-tuning adaptive vibration-based microgenerators," in Smart Materials, Nano-, and Micro-Smart Systems, pp. 373-384, International Society for Optics and Photonics, 2005 . 